\title{
Energy-Aware Sparse Approximation Technique (EAST) for Rechargeable Wireless Sensor Networks
}

\author{
Rajib Rana ${ }^{12}$, Wen $\mathrm{Hu}^{2}$, and Chun Tung $\mathrm{Chou}^{1}$ \\ ${ }^{1}$ School of Computer Sci. and Engineering, University of New South Wales, Australia \\ rajibr,ctchou@cse.unsw.edu.au \\ 2 CSIRO ICT Center, Australia. \\ wen.hu@csiro.au
}

\begin{abstract}
Due to non-homogeneous spread of sunlight, sensing nodes typically have non-uniform energy profiles in rechargeable Wireless Sensor Networks (WSNs). An energy-aware work load distribution is therefore necessary for good data accuracy while ensuring an energy-neutral operation. Recently proposed signal approximation strategies, in form of Compressive Sensing, assume uniform sampling and thus cannot be deployed to facilitate energy neutral operation in rechargeable WSNs. We propose a sparse approximation driven sensing technique (EAST) that adapts sensor node sampling workload according to solar energy availability. To the best of our knowledge, we are the first to propose sparse approximation for modeling energy-aware work load distribution in order to improve signal approximation from rechargeable WSNs. Experimental result, by using data from an outdoor WSN deployment, suggests that EAST significantly improves the approximation accuracy while supporting approximately $50 \%$ higher sensor on-time compared to an approach that assumes uniform energy profile of the nodes.
\end{abstract}

\section{Introduction}

Wireless Sensor Networks (WSNs) are currently deployed to monitor microclimate data from different environments $[1,21]$. The Springbrook National Park WSN is one such example. The Springbrook site is part of a World Heritage precinct in Queensland, Australia. CSIRO, in partnership with the Queensland Government Environmental Protection Agency (EPA), is in the process of deploying a WSN of 200 nodes at Springbrook by 2011 to collect micro-climate data for enhancing knowledge of rain forest restoration processes.

Energy supply is a major design constraint in the Springbrook deployment and the lifetime is limited by battery supplies. In the last few years, a large number of research has been conducted ([3] has a comprehensive list) to minimize the radio activities. However, recently it has been reported that many real life applications require specific sensors whose power consumption is significant [17].

\footnotetext{
${ }^{2}$ This work was done while Rajib Rana was an intern at CSIRO ICT center, Australia.
} 
Table 1. Energy consumption of some common radios [15, 21]. $T_{x}$ and $R_{x}$ are the transmission and the reception energy accordingly. We compute transmission energy for a 32 byte data packet.

\begin{tabular}{|c|c|c|}
\hline Radio & Producer & $\begin{array}{c}\text { Energy } \\
\text { Consumption }\end{array}$ \\
\hline CC2420 & Texas & $T_{x}: 34 \mu \mathrm{J}$ \\
& Instruments & $R_{x}: 38 \mu \mathrm{J}$ \\
\hline CC1000 & Texas & $T_{x}: 40 \mu \mathrm{J}$ \\
& Instruments & $R_{x}: 28 \mu \mathrm{J}$ \\
\hline
\end{tabular}

Table 2. Energy Consumption of some common sensors [21]. Sensors are turned on for 5 seconds for one reading (Sensors are turned on for 5 seconds every 5 minutes in the Springbrook deployment.).

\begin{tabular}{|c|c|c|}
\hline Sensor & Sensing & $\begin{array}{c}\text { Energy } \\
\text { Consumption }\end{array}$ \\
\hline Met One 034B & Wind Speed & $45 \mathrm{~mJ}$ \\
\hline Met One 034B & Wind Direction & $45 \mathrm{~mJ}$ \\
\hline
\end{tabular}

In addition, longer acquisition times of some specific sensors may even result in significantly higher energy consumptions than the radio (see Table 1 and 2 for a comparison of energy consumptions of some popular radio equipment with the energy hungry wind sensors). In order to cope with the increasing energy demand, a number of sensor deployments are adopting a complementary approach of supplementing the energy supply of the system by harvesting additional energy from the environment $[21,11]$.

Out of the variety of energy harvesting modalities, solar current harvesting provides one of the highest power densities [18]. However, solar energy will typically not be homogeneously spread over the network which results in nonhomogeneous energy profile (i.e non-uniform solar current harvest rates) of the sensing nodes. Therefore, sensing task allocation that assumes uniform energy profile of the sensing nodes could deplete the energy of a number of nodes and create holes in the network connectivity or coverage. In order to avoid such situation, the Springbrook deployment reduces the fraction of time the sensors are turned on to take samples (we refer this quantity as sensor on-time) to less than $2 \%$ for all nodes, which results in poor approximation of the signal.

Data collected from the wireless sensor deployments are typically correlated and therefore compressible [4] in an appropriate transform. Recent results in Compressive Sensing [6] suggests that if the data is compressible, a signal vector with $\hat{N}$ data values can be well approximated using only $k(<<\hat{N})$ transform coefficients. If the $k$ largest coefficients could be approximated from a small number of measurements, where measurements are taken with high probability from energy-rich sensing nodes and with smaller probability from energy-constrained nodes, we could approximate the signal with good accuracy while ensuring an energy neutral operation. An energy neutral operation means that the energy consumption should be less than the energy harvested from the environment. The estimation techniques of compressive sensing ([20, 4, 9]) have so far assumed that the signal is sampled uniformly. Therefore, in order to approximate a signal with good accuracy while ensuring an energy neutral operation, a theoretical framework that supports nonuniform sampling need to be developed. In this paper we address this challenge. Our contributions are as follows 
1. We present a distributed sensing framework, EAST, which for the first time implements sparse random projections to distribute sensing workload based on the solar energy harvest rates of the nodes to achieve an energy-neutral operation while at the same time is able to approximate a signal with good accuracy with high probability. Our work therefore draws a connection between compressive sensing and the sensor selection problem.

2. We determine the upper bound of sampling requirement of EAST as a function of $g_{j}$, which is a parameter that determines the sparsity of projection matrix and is proportional to the energy harvest rate of the sensing node $n_{j}$, and show that $O\left(\operatorname{poly}(k, \log \hat{N}) \sum_{j=1}^{N} \frac{1}{g_{j}}\right)$ sparse random projections are sufficient for EAST to reconstruct a signal with error, comparable to the best $k$-term approximation.

3. We evaluate EAST using the data collected from the Springbrook sensor deployment and report that energy-aware task distribution allows EAST to support approximately $50 \%$ higher sensor on-time, and thus allows EAST to achieve significantly better approximation compared to a sensing technique that assumes uniform energy profile of the nodes. Experimental result also reveals that EAST can achive approximation accuracy close to the best $k$ term approximation.

The remainder of the paper is organized as follows. In the next section, we precisely define EAST and describe the necessary modeling assumptions. Then we model EAST in Section 3 and describe a distributed algorithm for EAST in Section 4 . We provide the evaluation result in Section 5 and discuss the related literature in Section 6. Finally, we conclude in Section 7.

\section{Problem Definition}

Consider a signal $x$ captured over time $t_{h}, 1 \leq h \leq M$ from $N$ nodes $n_{j}$, $1 \leq j \leq N$ of a WSN. Assume that the network is rechargeable using solar energy. Define $E^{j}$ be the amount of energy harvested by node $n_{j}$ during time $t_{h}, 1 \leq h \leq M$ (in the rest of the paper we refer to $E^{j}$ as the energy profile of the node). Due to non-uniform spread of sunlight, $E^{j}$ can be non-uniform, e.g., nodes in the open space can have higher $E^{j}$ whereas nodes in the forest can have smaller $E^{j}$. We want to develop a sensing framework that distributes sampling workload based on $E^{j}$ (precisely we want the energy-rich sensors to work more and thus reduce the work load of energy-constrained sensors) and at the same time minimizes the approximation error while ensuring an energyneutral operation.

Let us further define an indicator variable

$$
f_{h j}= \begin{cases}1, & \text { if sensor } n_{j} \text { is turned on at } t_{h} \\ 0, & \text { otherwise. }\end{cases}
$$


In order to ensure an energy neutral operation, we turn on sensor $n_{j}{ }^{3}$ (i.e., $f_{h j}$ will be 1) based on its energy profile $E^{j}$. Consequently, some of the values of $f_{h j}$ could be zero. Note that the value of the signal $x$ at time instances where $f_{h j}=0$ are not measured, therefore, we need a method to compute an approximation of those components in $x$ that have not been measured. We aim to develop a method that achieves good approximation while maintaining energy-neutral operation.

In order to simplify the description, we will assume $M=1$ for the rest of this Section as well as in Section 3. This means that $x$ is a 1-dimensional vector and the $j$-th component of $x$ is in fact the sensor measurement of sensor $n_{j}$.

\subsection{Compressible Data}

Data collected from the wireless sensor deployments are typically correlated and therefore compressible in an appropriate transform [4]. Let us consider a transform $\Psi \in \mathbb{R}^{N \times N}$ (Wavelets or Discrete Fourier Transform are typically used as transforms), consisting of a set of orthonormal basis vectors $\left\{\psi_{1} . . \psi_{N}\right\}$. A signal $x$ is compressible, if the reordered transform coefficients $\theta=\left[\psi_{1}^{T} x, . ., \psi_{N}^{T} x\right]^{T}$ decay like power law [6], i.e., the $\pi$-th largest transform coefficient satisfies

$$
|\theta|_{(\pi)} \leq R \pi^{-\frac{1}{s}}
$$

for each $1 \leq \pi \leq N$, where $R$ is a constant, and $0 \leq s \leq 1$. We will call $s$ the compressibility parameter.

Recent results [6] of compressive sensing show that if the data is compressible, the largest (in magnitude) $k$ transform coefficients $(\theta)$ capture most of the signal information. A compressible signal can therefore be well approximated by recovering only the $k$ largest transform coefficients. The approximation that keeps the $k$ largest transform coefficients and discards the remaining as zero is called the best $k$-term approximation [20]. In order to model EAST we assume that the data collected at the energy-constrained nodes are correlated to the data collected at the energy-rich nodes and thus, if we collect large amount of data from the energy-rich nodes (and a small amount of data from energy constrained nodes), the $k$-largest coefficients could be recovered to have a good approximation of the signal.

\subsection{Sparse Random Projections}

In the literature it has been shown that if the signal is compressible, $\ell$ sparse random projections can be used to recover the signal with approximation error comparable to the best $k$-term approximation with high probabilities [20]. Unlike dense projection matrix (typically used in Compressive Sensing), the degree

\footnotetext{
${ }^{3}$ Note that, in WSN literature, a sensor can be used to refer to a sensor node (which includes a CPU, a radio and measurement sensors) or a measurement sensor (e.g. a temperature sensor, a wind speed sensor). In this paper, we refer to turning on sensor $n_{j}$ as to turning on the measurement sensor on node $n_{j}$
} 
of sparsity of the sparse random projections can control the number of measurements need to be acquired. For example, consider a sparse projection matrix $\Phi \in \mathbb{R}^{\ell \times N}$ with following entries.

$$
\Phi_{i j}=\sqrt{\rho} \begin{cases}+1 & \text { with prob. } \frac{1}{2 \rho} \\ 0 & \text { with prob. } 1-\frac{1}{\rho} \\ -1 & \text { with prob. } \frac{1}{2 \rho}\end{cases}
$$

$\rho$ determines the sparsity of the random projections. Thus, if $\frac{1}{\rho}=1$, the random matrix has no sparsity (i.e., it is dense); on the other hand, if $\frac{1}{\rho}=\frac{1}{N}$, the matrix is sparse and the expected number of non-zero elements in each row of the projection matrix is $1(=N / N)$.

In order to see how sparse projections can reduce the sampling requirement, let us first point out that the vector $u(=\Phi x)$ is required for signal estimation. Note that if for a particular value $j$, we have $\Phi_{i j}=0$ for all $i$ (in other words, the $j$-th column of $\Phi$ is all zero), then the $j$-th component of $x$ is not needed to obtain $u$. This means that node $n_{j}$ does not need to turn its sensor on to collect a sample. For the $\Phi_{i j}$ defined above, the mean number of sensors that are required to sample is given by $N\left(1-\left(1-\frac{1}{\rho}\right)^{\ell}\right)$, which can be showed to be bounded from above by $\frac{N \ell}{\rho}$. For $\rho=N$, this means at most $\ell$ samples are required. Since $\ell$ is supposed to be significantly less than $N$, the sampling requirement is low.

\section{Modeling EAST}

We use sparse random projections to model EAST. We control the sparsity of the projection matrix based on the energy profile such that measurements from energy rich sensing nodes are taken with high probabilities and those are taken from the energy constrained sensing nodes with small probabilities. Our sparse projection matrix $\Phi \in \mathbb{R}^{\ell \times N}$ has the following entries

$$
\Phi_{i j}=\sqrt{\frac{1}{g_{j}}} \begin{cases}+1 & \text { with prob. } \frac{g_{j}}{2} \\ 0 & \text { with prob. } 1-g_{j} \\ -1 & \text { with prob. } \frac{g_{j}}{2}\end{cases}
$$

Here $g_{j}=\frac{E^{j}}{\Sigma_{j=1}^{N} E^{j}} * \frac{\ell}{N}$ gives the probability of a measurement from sensor $n_{j}$ to be included in the $i$-th projection. Note that $g_{j}$ is proportional to the energy profile of node $n_{j}$, therefore higher energy profile of a node will increase the probability of inclusion of measurement from the node. If $\Phi_{i j} \neq 0$, we want measurement from sensor $n_{j}$ to be included in the $i$-th projection. In order to control sensor scheduling based on $\Phi_{i j}$, we determine the value of the indicator variable $f_{h j}$ based on the values of $\Phi_{i j}$. Let us consider one time snapshot $\left(t_{1}\right)$ of the data $\left(x_{1} \ldots x_{N}\right)$. Sensor scheduling to acquire this snapshot is determined by

$$
f_{1 j}= \begin{cases}0 & \text { if } \Sigma_{i=1}^{\ell}\left|\Phi_{i j}\right|=0 \\ 1 & \text { otherwise }\end{cases}
$$


We now prove that if the signal satisfies peak-to-total energy condition,

$$
\frac{\|x\|_{\infty}}{\|x\|_{2}} \leq \mu
$$

EAST approximates the signal with error comparable to the best $k$-term approximation with high probability. Note that the peak-to-total energy condition (5) can be related to the signal compressibility. If signal $x$ is compressible in a transform with compressibility parameter $s$, then the peak-to-total energy [20],

$$
\frac{\|x\|_{\infty}}{\|x\|_{2}} \leq \mu= \begin{cases}O\left(\frac{\log N}{\sqrt{(N)}}\right) & \text { if } s=1 \\ O\left(\frac{1}{\sqrt{(} N)}\right) & \text { if } 0<s<1\end{cases}
$$

We prove that EAST can approximate a signal with error comparable to the best $k$-term approximation in two stages. In the first stage (Proposition 1 ), we show that sparse random projections can produce estimation for the transform coefficients of the data. Then in the second stage (Proposition 2), we show that the approximation error of the estimation is comparable to the best $k$-term approximation.

Note that the transform coefficients of the data are the inner product between the data and the set of orthonormal bases. Therefore, we first show that sparse random projections of our projection matrix preserve inner products within a small error, with high probability. Proposition 1 states that an estimation of the inner product between two vectors, using only the random projections defined by Equation (3), has the correct expectation with bounded variance (The proof of Proposition 1 is shown in Appendix).

Proposition 1 Let $\Phi$ be the projection matrix given by Equation (3). Define $u=\frac{1}{\sqrt{\ell}} \Phi x$ and $v=\frac{1}{\sqrt{\ell}} \Phi y \in \mathbb{R}^{\ell}$ as the random projection of two vectors $x$ and $y \in \mathbb{R}^{\ell}$. Expectation and variance of the inner product of $u$ and $v$ are respectively

$$
\begin{aligned}
\mathbb{E}\left[u^{T} v\right] & =x^{T} y \quad \text { and } \\
\operatorname{Var}\left(u^{T} v\right) & =\frac{1}{\ell}\left(\left(x^{T} y\right)^{2}+\|x\|_{2}^{2}\|y\|_{2}^{2}+\Sigma_{j=1}^{N} \frac{1}{g_{j}} x_{j}^{2} y_{j}^{2}-3 \Sigma_{j=1}^{N} x_{j}^{2} y_{j}^{2}\right) .
\end{aligned}
$$

It can be observed that the variance of the estimation is largely controlled by the factor $\Sigma_{j=1}^{N} \frac{1}{g_{j}}$. Thus, if $g_{j}$ is a small value for a node $n_{j}$, the estimation will have high variance. Note that $g_{j}$ is proportional to the energy profile $E^{j}$, therefore when all the nodes have good access to sunlight, good estimation can be produced. Apart from $\Sigma_{j=1}^{N} \frac{1}{g_{j}}$, the variance of the estimation is also significantly controlled by the number of projections. A large value of $\ell$ could produce a smaller variance. In Proposition 2, we will determine the value of $\ell$ based on the factor $\Sigma_{j=1}^{N} \frac{1}{g_{j}}$. Note that in [20] it is shown that the variance of this estimation is controlled by the number of projections $(\ell)$ only and it is not shown that how the variance will be changed if the nodes have non-uniform energy profile. 
Having showed that the estimation of the inner product between two vectors, using only the sparse projections of those vectors, has a good quality estimation with bounded variance, it can be shown (see Lemma 71 in Appendix) that the error of the estimation $\hat{a}_{i}$ for $x^{T} y_{i}$, using the sparse random projections $\frac{1}{\sqrt{\ell}} \Phi x$ and $\frac{1}{\sqrt{\ell}} \Phi y_{i}$, satisfies

$$
\left|\hat{a_{i}}-x^{T} y_{i}\right| \leq \epsilon|| x\left\|_{2}\right\| y_{i} \|_{2}, \forall_{i=1 . . N}
$$

Finally, in Proposition 2 we state that the estimation error determined in Equation (7) is comparable to the $k$-term approximation with high probability (proof is included in Appendix).

Proposition 2 Assume data $x \in \mathbb{R}^{N}$ satisfies the peak-to-total energy condition (5), and with

$$
\ell=O\left(\frac{1+\gamma}{\epsilon^{2} \eta^{2}} k^{2} \mu^{2} \log N \Sigma_{j=1}^{N} \frac{1}{g_{j}}\right)
$$

the sparse random matrix $\Phi \in \mathbb{R}^{\ell \times N}$ satisfies condition (9). Denote $u=\frac{1}{\sqrt{\ell}} \Phi x$ as the sparse random projection of $x$ and $\Psi \in \mathbb{R}^{N \times N}$ as an orthonormal transform. Transform coefficients of $x$ in $\Psi$ is given by, $\theta=\Psi^{-1} x$. Assume the best $k$-term approximation gives an approximation $\left(\hat{x}_{\text {opt }}\right)$ with error $\left\|x-\hat{x}_{\text {opt }}\right\|_{2}^{2} \leq \eta\|x\|_{2}^{2}$. Using only $u, \Phi$ and $\Psi, x$ can be recovered with error

$$
\frac{\|x-\hat{x}\|_{2}^{2}}{\|x\|_{2}^{2}} \leq(1+\epsilon) \eta
$$

with probability at least $1-N^{-\gamma}$.

From Proposition 2 it can be observed that a smaller value of the peak-to-total energy $(\mu)$ makes the requirement of number of projections $\ell$ to be small (this is inherent to sparse approximation). However, $\ell$ is largely controlled by the factor $\Sigma_{j=1}^{N} \frac{1}{g_{j}}$. Thus, if $g_{j}$ is a small value for a node $n_{j}$, a large number of projections is required to achieve an accuracy similar to the best $k$-term approximation. One of the main contributions of our work is that we enable energy-aware work load allocation and thus support a large $\ell$ to achieve an accuracy comparable to the best $k$-term approximation.

\section{Distributed Algorithm}

Energy-aware work load allocation typically increases the amount of communications between node and the base station and thus increases the consumption of transmission energy. We therefore design a distributed algorithm for EAST, which generates projections with a few communications between the sensing nodes and the base station. 
Note that our description so far has assumed $M=1$, however the framework can be readily extended to the case with $M>1$. In this case, we consider the sensor measurement $x_{h j}$ collected at time $t_{h}(h=1, \ldots, M)$ by sensor $n_{j}(j=$ $1, . ., N)$. Since the algorithm in Section 3 works with a vector, we will vectorize the 2-dimensional signal $x_{h j}$. We will abuse the notation and use $x$ to denote this vector (this should be clear from the context). The vector $x$ has $\hat{N}=M N$ elements where the $q$-th element of $x$ is $x_{h j}$ where $q=h+(j-1) * M$. The corresponding projection matrix $\Phi$ is now an $\ell \times \hat{N}$ matrix. For $q=h+(j-1) * M$, the elements in the $q$-th column of the projection matrix $\left(\Phi_{i q}\right.$ with $\left.i=1, \ldots, \ell\right)$ are generated by Equation (3) with parameter $g_{j}$ and these elements will determine whether the sensor $n_{j}$ will sample at time $t_{h}$. We will now describe an algorithm which is used by EAST to recover an approximation of the signal $(x)$, from the sparse projections created locally in different nodes.

- Initially, sensor node $n_{\tilde{j}}(1 \leq \tilde{j} \leq N)$ locally decides to generate $\ell_{\tilde{j}}$ rows of the projection matrix where $0 \leq \ell_{\tilde{j}} \leq \ell$ and $\sum_{\tilde{j}=1}^{N} \ell_{\tilde{j}}=\ell$.

- Then each node $n_{\tilde{j}}(1 \leq \tilde{j} \leq N)$ generates the random numbers $\Phi_{r 1}, \ldots, \Phi_{r \hat{N}}$ using the distribution function mentioned in Equation(3)(We assume that node $n_{\tilde{j}}$ is responsible for generating the $r$-th row $(1 \leq r \leq \ell)$ of the projection matrix. Consider the element $\Phi_{r q}$ in the projection matrix and let us assume that the column index $q$ and the node-time pair $(j, h)$ have one-to-one correspondence given by $q=(j-1) * M+h$.).

- If $\Phi_{r q} \neq 0$, node $n_{\tilde{j}}$ tasks node $n_{j}$ to sample at time $t_{h}$ and node $n_{j}$ sends the sample to node $n_{\tilde{j}}$.

- Upon receiving $x_{j h}$ from node $n_{j}, n_{\tilde{j}}$ computes $u_{r}=\Sigma_{q=1}^{\hat{N}} \Phi_{r q} x_{q}$ (where $x_{q}=x_{j h}$ ). Node $n_{\tilde{j}}$ performs this operation for all the values it receives and finally transmits $u_{r}$ to the base station. This process is repeated for all node $n_{\tilde{j}}, 1 \leq \tilde{j} \leq N$.

- After receiving transmissions from the nodes, base station has $\Phi_{\ell \times \hat{N}} x=$ $\left[u_{1}, \ldots, u_{\ell}\right]^{T}$. It then generates $\Phi_{\ell \times \hat{N}}$ using the same seed as the nodes. Finally, with $u\left(=\Phi_{\ell \times \hat{N}} x\right), \Phi_{\ell \times \hat{N}}$ and $\Psi$, base station uses AMS sketching decoder [2] to recover the signal.

\section{Evaluation}

In this Section we evaluate the performance of EAST using a number of microclimate data such as wind speed, wind direction, soil moisture and air temperature, collected from the Springbrook sensor deployment. We carefully choose the micro-climate data to evaluate EAST using both high and low frequency signals. We have used data from 8 of the sensing nodes at the Belmont deployment, where among these 8 nodes (shown in Fig. 1(a)), node 5 is deep in the forest whereas the rest of the nodes are in the open space. Consequently, solar current harvest rate of node 5 is the lowest whereas the rest of the nodes have higher and also similar harvest rates (see Fig. 1(b)). Inter-sampling interval in 


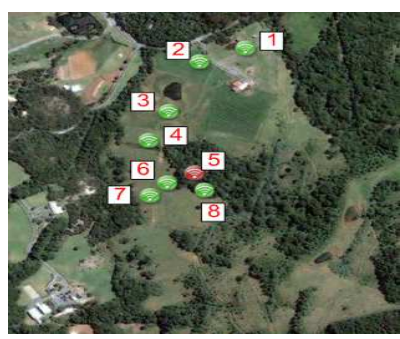

(a) Positions of the sensing nodes at Springbrook deployment. Node 5 is in the deep forest whereas rest of the nodes are mostly in the open space.

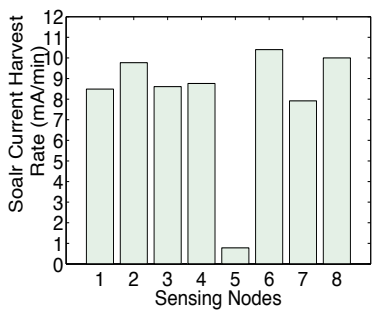

(b) Energy profile of the sensing nodes at Springbrook deployment

Fig. 1. Location and energy harvesting rate of the Springbrook sensing nodes

the deployment is 5 minutes and we collected 1 month data which gives us 7680 snapshots for both of all the four micro-climate data. As AMS sketching decoder computes the estimation from median, it performs better with large $\hat{N}$. Therefore, we have arbitrarily chosen large $\hat{N}=2048$. We made 30 smaller datasets from 7680 snapshots, where each set has $M=256$ snapshots from $N=8$ nodes. Thus, we get $\hat{N}=M N=2048$. Note that we have also tested that for other large values of $\hat{N}$, such as 512 and 1024, EAST produces similar approximation.

\subsection{Uniform-Energy Sensing Technique, UEST}

We compare the performance of EAST with a uniform energy sensing technique (UEST). UEST assumes that nodes have homogeneous energy profile and therefore allocates sampling workload uniform randomly. In particular we use EAST to create UEST where we deliberately modify the energy profile $E^{j}$ of all sensing nodes $1 \leq j \leq N$ to be equal (We use equal energy profile $\hat{E}^{j}=1 / 8 \Sigma_{1 \leq j \leq N} E^{j}$ ). In addition to providing a way to compare EAST with a sensing technique which assumes uniform energy in all sensing nodes, UEST also facilities the evaluation of EAST at uniform energy condition.

\subsection{Approximation Error}

Let $\hat{x}$ be the approximation of the signal $x$, we use relative error, $\|x-\hat{x}\|_{2}^{2} /\|x\|_{2}^{2}$ to determine the accuracy of the approximation. The relative error is a commonly used error metric in the signal processing literature $[12,20]$ that tells us how close the approximate signal is to the real signal.

\subsection{Results}

Peak-to-total energy condition is a sufficient condition for sparse approximation. In Fig. 2 we find that for both of the wind data $\frac{\|x\|_{\alpha}}{\|x\|_{2}}$ is bounded by $\frac{\log \hat{N}}{\sqrt{\hat{N}}}$ and 


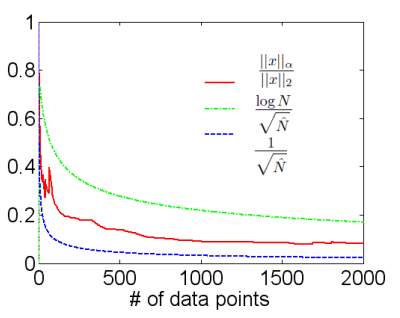

(a) Wind Speed

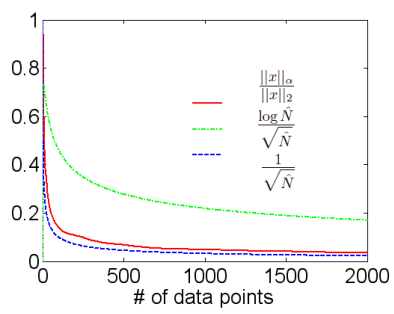

(b) Wind Direction

Fig. 2. Peak-to-total energy condition on data.

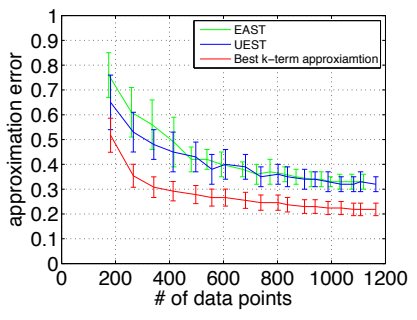

(a) Wind Speed

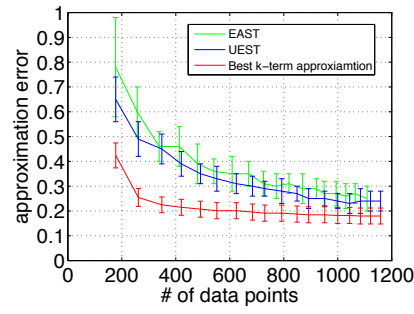

(b) Wind Direction

Fig. 3. A comparison of the approximation error of the micro-climate data using EAST, UEST, and optimal Haar wavelet based approximation. The relative approximation error is plotted versus the number of random projections $\ell=k^{2} \log \hat{N}$ for $\hat{N}=2048$. The error bars show the standard deviation of the approximation error.

$\frac{1}{\sqrt{\hat{N}}}$. Therefore, according to Equation (6) the wind sensor data obey the peakto-total energy condition. From Fig. 2 we also observe that the compressibility parameter $s$ is bounded by $0<s \leq 1$, therefore, the wind sensnor data are also compressible (see Equation (2)).

Fig. 3 compares the approximation accuracy of EAST and UEST against different number of data points. We vary the number of projections $(\ell)$ and extract the number of data points included in the $\ell$ projections and then plot the approximation accuracy against the number of data points to precisely demonstrate the sensing requirements of EAST. For each number of data points, we use the snapshots collected from the Springbrook deployment to compute the mean and standard deviation of the approximation error. We observe that unless for very small number of data points, the mean and the standard deviation of the approximation error using EAST is as good as UEST. Precisely, by using $400(=19 \%)$ data points, EAST achieves an approximation error below 0.5. Fig. 3 also compares the approximation of EAST and UEST with the best $k$ - 


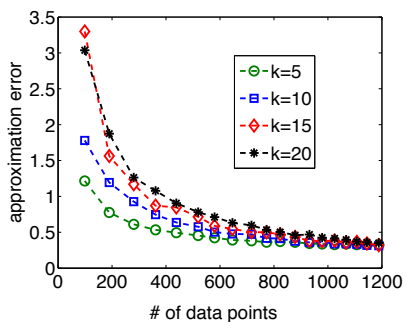

(a) Wind Speed

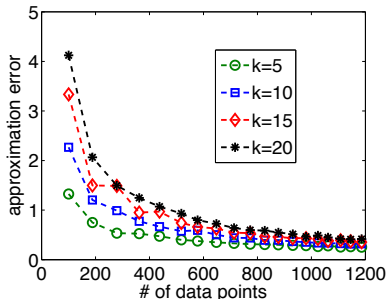

(b) Wind Direction

Fig. 4. Impact of the number of significant coefficients retained $(k)$ on the approximation of EAST. The relative approximation error is plotted versus the number of sampled data points for different value of $k$. Reconstruction is poor for $k=1$ and thus is excluded from the figure.

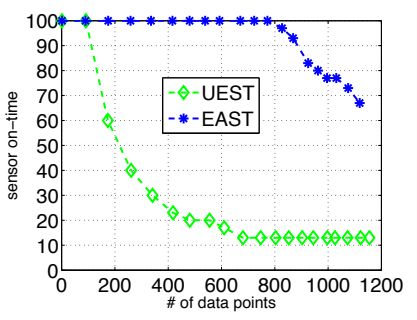

(a) Wind Speed

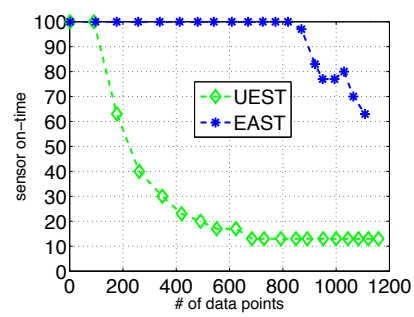

(b) Wind Direction

Fig. 5. Comparison of sensor on-time supported by EAST and UEST at energy neutral condition. The relative approximation error of the data is plotted versus the number of sampled data points for $\hat{N}=2048$.

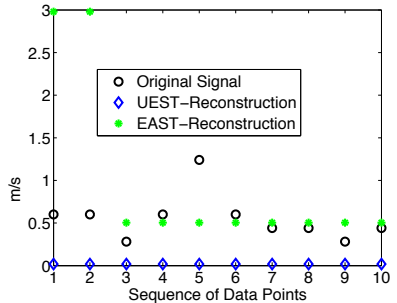

(a) Wind Speed

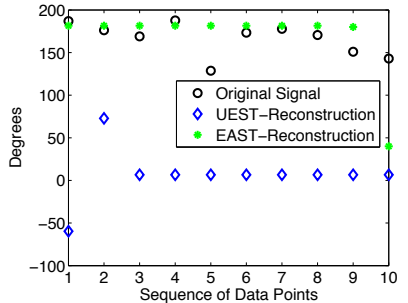

(b) Wind Direction

Fig. 6. (a) Recovery of temporal signal at node 5. In order to avoid cluttering of image we show only 10 data points of the signal. Similar recovery is observed for rest of the data points. 
term approximation. We observe that both EAST and UEST performs closer to the best $k$-term approximation when the number of data points are more than 800 .

Using sparse approximation, we only reconstruct the significant coefficients of a signal in the transform domain and let the insignificant coefficients to be zero. We have investigated the impact of the number of significant coefficients being retained $(k)$ on the accuracy of approximation and found that, unless using very small value (e.g. $k=1$ ), accuracy of the approximation is similar for different values of $k$. However, in order to avoid cluttering the images, in Fig. 4, we plot $\frac{\|x-\hat{x}\|_{2}^{2}}{\|x\|_{2}^{2}}$ versus $k$ for $k$ up to 20 . We use $k=5$ for the rest of the paper.

One of the major contributions of this paper is, EAST attempts to minimize approximation error by increasing sensor on-time. Here we use the term sensor on-time to indicate the fraction of time a sensor is on when it takes a sample (For example, for an inter-sampling period of 5 minutes and a sensor on-time of 0.6 , the sensor will be turned on for $0.6^{*} 5=3$ minutes every time the sensor takes a sample. Note that it can be shown that the duty cycle of a sensor is given by the product of its sampling probability and sensor on-time.). The sensor ontime is common for the network, however, the sampling probability of a sensor is determined by its energy profile. In order to show that EAST supports longer sensor on-time, we compare the maximum sensor on-time (while maintaining an energy neutral operation) supported by EAST and UEST for different number of data points. Note that in the Springbrook deployment, battery voltage $V=3$ Volts, the electrical current used to acquire a wind sample is $I=3 \mathrm{~mA}$ and the inter-sampling interval is $T=5$ minutes (300 seconds). Therefore, if the sensor on-time is $\omega$, then the amount of energy spent by sensor $n_{j}$ over the period $t_{h}$ (where $h=1, \ldots, M$ ) is given by $\Delta_{j}=V I \omega T \Sigma_{1 \leq h \leq M} f_{h j}$. The maximum sensor on-time that is supported by the network is the maximum value of $\omega$ such that $\Delta_{j} \leq E^{j}$ for all $j$.

In Fig. 5 we compare supported sensor on-time for different number of data points. It is observed that, when we use $1200(<50 \%)$ data points, EAST can support $50 \%$ longer sensor on-time compared to UEST.

Now let us show the impact of sensor on-time on the accuracy of approximation. In Fig. 6 we plot the approximated signal along with the real signal collected in node 5 . We choose node 5 deliberately to show the robustness of EAST. Note that node 5 has the lowest energy profile and therefore has the least sampling probability. We use 1200 data points and sensor on-time to be 0.5 for this approximation. While using UEST, node 5 fails due to exceeding its energy budget, which causes poor approximation, whereas energy-aware workload distribution yields significantly better approximation for EAST.

\section{Related Work}

A large number of signal approximation techniques use Compressive Sensing [7, $20,4,9]$ to conserve transmission energy assuming that radio is the dominant 
component of energy consumption, however we assume energy-hungry sensor dominates the energy consumption.

In [5] an adaptive sampling algorithm is presented which can be used for estimating the best sampling frequency for energy hungry sensors. However, similar to the work of compressive sensing $[20,4,9]$ their approach assume that the sensors have uniform energy profiles.

Work presented in [13] proposes a harvest-aware adaptive sampling approach to dynamically identify the maximum duty cycle. However, their focus is not on signal approximation from the network.

An application-specific approach for energy conservation is presented in [23] where adaptive sampling and energy-aware routing are applied jointly to recover a signal. However, we consider energy-aware data acquisition in our paper.

In [19], a Bayesian estimation technique is presented to estimate the wind speed and wind direction signals. They have supplemented their estimation using the assumption that the wind speed and wind direction signals have a correlation with hourly tide data. However, in our work we assume that signals are compressible due to the presence of spatial-temporal correlation among the data collected at different sensing nodes.

A number of studies $[14,22,10,8]$ have proposed to exploit the spatialtemporal correlation of the signal to reduce sampling requirements. Though our approach has similar assumption, we have considered non-uniform energy profile of the sensors which is different. Moreover, we have used Sparse Approximation which is also different from their approaches.

A Compressive Sensing based data gathering approach is presented in [16] which investigates the impact of a routing topology generated sparse projection matrix on the accuracy of the approximation. Our work is different from theirs since our projection matrix is not based on the routing topology rather it is populated based on the energy profile of the sensors.

\section{Conclusion}

This paper proposes an energy-aware sensing technique (called EAST) that implements distributed sparse random projections to adapt sampling workload distribution based on the solar energy availability at nodes, and thus recovers an approximation of the signal with good accuracy while ensuring an energy neutral operation. A large number of recently developed compressive sensing driven approximation strategies assume that each element of the projection vector is drawn from the same probability distribution. This inherently assumes uniform sampling and thus is inapplicable for ensuring energy neutral operation when nodes have non-uniform energy profiles.. We develop a theoretical framework to determine the number of projections need to be collected as a function of the energy profile of the nodes and prove that $O\left(\operatorname{poly}(k, \hat{N}) \Sigma_{j=1}^{\hat{N}} \frac{1}{g_{j}}\right)$ sparse projections are sufficient to reconstruct a $\hat{N}$ data point signal with accuracy comparable to the best $k$-term approximation. We apply EAST to reduce the energy consumption of wind speed and wind direction sensors; however, EAST is general and 
can be used for any signal that satisfies the peak-to-total energy condition. Evaluation result shows that EAST increases the sensor on-time by approximately $50 \%$ and thus offers significantly better approximation of a signal compared to a sensing technique that assumes uniform energy profile of nodes. Experimental result also supports that approximation accuracy of EAST is close to the best $k$-term approximation.

\section{References}

[1] Habitat monitoring on great duck island. http://www.greatduckisland.net/index.php.

[2] Noga Alon, Yossi Matias, and Mario Szegedy. The space complexity of approximating the frequency moments. In JOURNAL OF COMPUTER AND SYSTEM SCIENCES, pages 20-29, 1996.

[3] G. Anastasi, M. Conti, M. Francesco, and A. Passarella. Energy conservation in wireless sensor networks: A survey. Ad Hoc Networks, 7(3):537-568, May 2009.

[4] Waheed Bajwa, Jarvis Haupt, Akbar Sayeed, and Robert Nowak. Compressive wireless sensing. In IPSN'06, pages 134-142, 2006.

[5] C.Alippi, G. Anastasi, M. D. Francesco, and M. Roveri. An adaptive sampling algorithm for effective energy management in wireless sensor networks with energyhungry sensors. In IEEE-Transactions on Instrumentation and Measurement.

[6] E. Candés. Compressive sampling. In Proc. of the Int. Congress of Mathematics, 2006.

[7] Chun Tung Chou, Rajib Rana, and Wen Hu. Energy efficient information collection in wireless sensor networks using adaptive compressive sensing. In In Proc. of the 34th Annual IEEE Conference on Local Computer Networks (LCN 2009), pages 443-450, 2009.

[8] Amol Deshpande, Carlos Guestrin, Sam Madden, Joseph Hellerstein, and Wei Hong. Model-driven data acquisition in sensor networks. In $V L D B$ ' 04 , pages 588-599. VLDB Endowment, 2004.

[9] Marco F. Duarte, Michael B. Wakin, Dror Baron, and Richard G. Baraniuk. Universal distributed sensing via random projections. In IPSN '06, pages 177$185,2006$.

[10] Himanshu Gupta, Vishnu Navda, Samir Das, and Vishal Chowdhary. Efficient gathering of correlated data in sensor networks. ACM Trans. Sen. Netw., 4(1):1$31,2008$.

[11] Wen Hu, Nirupama Bulusu, Chun Tung Chou, Sanjay Jha, Andrew Taylor, and Van Nghia Tran. Design and evaluation of a hybrid sensor network for cane toad monitoring. ACM Trans. Sen. Netw., 5(1):1-28, 2009.

[12] Shihao Ji, Ya Xue, and Lawrence Carin. Bayesian compressive sensing. IEEE Trans. Signal Processing, 2007.

[13] Aman Kansal, Jason Hsu, Sadaf Zahedi, and Mani B. Srivastava. Power management in energy harvesting sensor networks. Trans. on Embedded Computing Sys., $6(4): 32+, 2007$.

[14] Chong Liu, Kui Wu, and Jian Pei. An energy-efficient data collection framework for wireless sensor networks by exploiting spatiotemporal correlation. IEEE Trans. Parallel Distrib. Syst., 18(7):1010-1023, 2007.

[15] Joseph Robert Polastre. PhD thesis.

[16] Giorgio Quer, Riccardo Masiero, Daniele Munaretto., Michele Rossi, Joerg Widmer, and Michele Zorzi. On the interplay between routing and signal representation for compressive sensing in wireless sensor networks. In ITA, 2007. 
[17] V. Raghunathan, S. Ganeriwal, and M. Srivastava. Emerging techniques for long lived wireless sensor networks. Communications Magazine, IEEE, 44(4):108-114, 2006.

[18] Vijay Raghunathan, Aman Kansal, Jason Hsu, Jonathan Friedman, and Mani B. Srivastava. Design considerations for solar energy harvesting wireless embedded systems. In IPSN '05, page 64, 2005.

[19] S. Reboul and M. Benjelloun. Joint segmentation of the wind speed and direction. Signal Process., 86(4):744-759, 2006.

[20] Wei Wang, Minos Garofalakis, and Kannan Ramchandran. Distributed sparse random projections for refinable approximation. In IPSN ' $0 \%$, pages 331-339, 2007.

[21] T. Wark, Wen Hu, P. Corke, J. Hodge, A. Keto, B. Mackey, G. Foley, P. Sikka, and M. Brunig. Springbrook: Challenges in developing a long-term rainforest wireless sensor network. In ISSNIP, Dec. 2008.

[22] Rebecca Willett, Aline Martin, and Robert Nowak. Backcasting: adaptive sampling for sensor networks. In IPSN'04, pages 124-133, 2004.

[23] Jing Zhou and David De Roure. Floodnet: Coupling adaptive sampling with energy aware routing in a flood warning system. J. Comput. Sci. Technol., 22(1):121130, 2007.

\section{APPENDIX}

Proof (Proof of Proposition 1.). It can be proved that the projection matrix defined by Equation (3) satisfies these conditions:

$$
\mathbb{E}\left[\Phi_{i j}\right]=0, \mathbb{E}\left[\Phi_{i j}^{2}\right]=1, \mathbb{E}\left[\Phi_{i j}^{4}\right]=\frac{1}{g_{j}} .
$$

Define independent random variables $w_{1}, . . w_{\ell}$ where, $w_{i}=\left(\sum_{j=1}^{N} x_{j} \Phi_{i j}\right)\left(\sum_{j=1}^{N} y_{j} \Phi_{i j}\right)$ Using the property in Equation (9), it can be shown that the expectation and the second moment of $w_{i}$ satisfy: $\mathbb{E}\left[w_{i}\right]=x^{T} y$ and

$$
\mathbb{E}\left[w_{i}^{2}\right]=2\left(x^{T} y\right)^{2}+\|x\|_{2}^{2}\|y\|_{2}^{2}+\Sigma_{j=1}^{N} \frac{1}{g_{j}} x_{j}^{2} y_{j}^{2}-3 \Sigma_{j=1}^{N} x_{j}^{2} y_{j}^{2} .
$$

Since $u^{T} v=\frac{1}{\ell} \sum_{i=1}^{\ell} w_{i}$, using the above result, we can show that:

$$
\operatorname{Var}\left(u^{T} v\right)=\frac{1}{\ell}\left(\left(x^{T} y\right)^{2}+\|x\|_{2}^{2}\|y\|_{2}^{2}+\sum_{j=1}^{N} \frac{1}{g_{j}} x_{j}^{2} y_{j}^{2}-3 \Sigma_{j=1}^{N} x_{j}^{2} y_{j}^{2}\right) .
$$

In order to prove proposition 2, we need the following lemma.

Lemma 71 Consider a data vector $x \in \mathbb{R}^{N}$ which satisfies condition (5). Let $y \in \mathbb{R}^{N \times N}$. Consider a sparse random matrix $\Phi \in \mathbb{R}^{\ell \times N}$ satisfies condition (9), with sparsity parameter $\rho=g_{j}$. Define $\ell=O\left(\frac{1+\gamma}{\epsilon^{2}} \mu^{2} \log N \Sigma_{j=1}^{N} \frac{1}{g_{j}}\right)$. The random projections $\frac{1}{\sqrt{\ell}} \Phi u$ and $\frac{1}{\sqrt{\ell}} \Phi v_{i}$ then produces an estimation $\hat{a}_{i}$ for $x^{T} y_{i}$, with probability at least $1-N^{-\gamma}$, satisfying $\left|\hat{a}_{i}-x^{T} y_{i}\right| \leq \epsilon\|x\|_{2}\left\|y_{i}\right\|_{2}, \forall_{1 \leq i N}$

Proof (Proof of Lemma 71). Due to lack of space, the proof for Lemma 71 cannot be included. The proof is similar to that of Theorem 1 in [20] except that the term $(s-3)$ in [20] is replaced by $\left(\sum_{j=1}^{N} \frac{1}{g_{j}}-3\right)$. 
Proof (Proof of Proposition 2). Consider an orthonormal transform $\Psi \in \mathbb{R}^{N \times N}$. Let the transform coefficients $\theta=\left[x^{T} \psi_{1}, \ldots, x^{T} \psi_{N}\right]^{T}$. Let us order the transform coefficients $\theta$ in decreasing of magnitude, i.e., $|\theta|_{(1)} \geq|\theta|_{(2)} \ldots \geq|\theta|_{(N)}$. The approximation error by taking the largest $k$ coefficients in magnitude, and setting the remaining coefficients to zero can therefore be given by $\left\|\theta-\hat{\theta}_{\text {opt }}\right\|_{2}^{2}=$ $\Sigma_{i=k+1}^{N}|\theta|_{(i)}^{2}$. Let $\left\|\theta-\theta_{\text {opt }}\right\|_{2}^{2} \leq \eta\|\theta\|_{2}^{2}$ and assume that $x$ satisfies condition (5), with positive integer, $\ell=O\left(\frac{1+\gamma}{\beta^{2}} \mu^{2} \log N \Sigma_{j=1}^{N} \frac{1}{g_{j}}\right)$. The random projections $\frac{1}{\sqrt{\ell}} \Phi u$ and $\left\{\frac{1}{\sqrt{\ell}} \Phi \psi_{1}, \ldots, \cdot \frac{1}{\sqrt{\ell}} \Phi \psi_{n}\right\}$ thus could produce estimates $\left\{\hat{\theta_{1}}, \ldots, \hat{\theta_{N}}\right\}$, where the estimates satisfy $\left|\hat{\theta}_{i}-\theta_{i}\right| \leq \beta\|\theta\|_{2}$ with high probability (Lemma 71).

Now ordering the estimates $\hat{\theta}$ in decreasing magnitude, we define our approximation $\tilde{\theta}$ as keeping the $k$ largest (in magnitude) components of $\hat{\theta}$, and setting the other components to zero. It can be shown that [20] for $\beta=O\left(\frac{\epsilon \eta}{k}\right)$, the approximate error is $\|x-\hat{x}\|_{2}^{2}=(1+\epsilon) \eta\|x\|_{2}^{2}$. Therefore the number of random projections we need can be given by

$$
\ell=O\left(\frac{1+\gamma}{\epsilon^{2} \eta^{2}} k^{2} \mu^{2} \log N \Sigma_{j=1}^{N} \frac{1}{g_{j}}\right)
$$

